\title{
Gênese do Laboratório de Física da Universidade Federal do Pará
}

\author{
Genesis of the Physics Laboratory of the Federal University of Pará \\ Luís Carlos Bassalo Crispino*10, Victor Façanha Serra ${ }^{1}$ \\ ${ }^{1}$ Universidade Federal do Pará, Faculdade de Física, Belém, Pará, Brasil
}

Recebido em 21 de Fevereiro, 2018. Revisado em 21 de Março, 2018. Aceito em 09 de Abril, 2018.

\begin{abstract}
Neste artigo tratamos do processo de instalação, composição inicial de equipamentos e capacitação de pessoal do Laboratório de Física da Universidade Federal do Pará, com ênfase nos experimentos recebidos da Alemanha, na década de 1970.

Palavras-chave: Laboratório de Física, Universidade Federal do Pará.
\end{abstract}

We report on the process of installation, initial equipment composition and staff training of the Physics Laboratory of the Federal University of Pará, with emphasis on the experiments received from Germany, in the 1970 decade.

Keywords: Physics Laboratory, Federal University of Pará.

Este artigo trata da instalação do Laboratório de Física da Universidade Federal do Pará (UFPA) na década de 1970, com ênfase na obtenção dos experimentos provenientes da Alemanha, assim como das iniciativas para capacitação de pessoal, objetivando o uso destes experimentos no Curso de Graduação em Física da UFPA.

A então denominada Universidade do Pará, integrada ao Ministério da Educação e Cultura (Diretoria de Ensino Superior), foi criada em 1957, tendo Juscelino Kubitschek de Oliveira como Presidente da República do Brasil [1,2].

O primeiro exame de acesso (vestibular) a um Curso Superior de (Licenciatura em) Física no Pará foi realizado em 1965, sob responsabilidade do Núcleo de Física e Matemática (NFM) [3], cujas atividades acadêmicas iniciaram em 1961 [4,5].

Para compor o acervo do laboratório do NFM, foram trazidos alguns experimentos relacionados à física da Escola de Engenharia 6,7,.11 Entre estes experimentos, estavam Hemisférios de Magdeburgo (cf. Fig. S1 - Material Suplementar), Centrífuga (cf. Fig. S2 - Material Suplementar), Câmara Escura (cf. Fig. S3 - Material Suplementar) e Bobina de Ruhmkorff (cf. Fig. S4 - Material Suplementar). $\mathrm{L}^{2} \mathrm{~L}^{3}$

\footnotetext{
*Endereço de correspondência: crispino@ufpa.br.

${ }^{1}$ Informação confirmada em entrevista concedida por Fernando Medeiros Vieira a Luís C. B. Crispino.

2 Alguns destes equipamentos foram vendidos para as instituições do Pará pelo estabelecimento comercial Otto Bender, com sede na cidade de São Paulo. Destacamos ainda um experimento ilustrativo de um Motor a Combustão (cf. Fig. S5 - Material Suplementar) que tem em sua etiqueta de tombamento a inscrição Universidade do Pará, sob o número 419, sendo portanto um dos primeiros 500 itens incorporados ao patrimônio da instituição.

3 Compondo a primeira turma de estudantes do Curso de (Licenciatura em) Física da UFPA, havendo ingressado em 1965 e com
}

Os equipamentos didáticos de física do NFM foram incorporados ao acervo do Laboratório de Física da UFPA, ${ }^{4}$ cujo prédio, já no Campus Universitário do Guamá (então denominado Conjunto Pioneiro ou Núcleo Pioneiro), foi inaugurado em 22 março de 1971 [8 10. Na ocasião daquela inauguração, veio a Belém o então Ministro da Educação e Cultura, Jarbas Gonçalves Passarinho, conforme anunciado pela imprensa paraense [11:

"O ministro Jarbas Passarinho estará proferindo a aula inaugural da Universidade Federal do Pará, às 10 horas de amanhã [22 de março de 1971], marcando a implantação da reforma universitária deste Estado. A aula [será] dada no Conjunto Pioneiro do Guamá, com a presença de calouros, veteranos, professores e funcionários da Universidade Federal do Pará."

Em seu discurso daquele dia, publicado pela imprensa local, o então Reitor da UFPA, Aloysio da Costa Chaves registrou [9]:

"[...] Empreendemos ainda, em 1970, o planejamento total do Conjunto Pioneiro do Guamá [...]. [...] demos ênfase à construção dos grandes laboratórios para o ensino básico de Química, Geologia, Física e Biofísica, que hoje serão também inaugurados, aparelhando-os em parte com precioso equipamento obtido com convênio, pelo MEC, da República Democrática da Alemanha [sic.]."

Algum tempo depois a UFPA recebeu um vasto conjunto de equipamentos da então República Federal da

colação de grau em 1968, estavam Ana Emília Coelho de Souza Bastos, Carmelina Nobukó Kobayashi, e José Maria Costa de Souza 3 . Carmelina Kobayashi nos relatou que durante o seu curso de Licenciatura em Física da UFPA as atividades experimentais foram bastantes restritas.

${ }^{4}$ Informação confirmada em entrevista concedida por Fernando Medeiros Vieira a Luís C. B. Crispino. 
Alemanha (RFA), no âmbito de um convênio entre o à época denominado Conselho Nacional de Pesquisa (CNPq), pelo lado brasileiro, e o KernForschungsAnlage (KFA), pelo lado alemão. Neste contexto, no ano de 1972, esteve no Brasil Heinz S. Räde, vindo da Alemanha com o objetivo de visitar algumas instituições brasileiras que receberiam os equipamentos alemães, incluindo as Universidades Federais do Pará, Ceará, Bahia e Santa Catarina 12 5

Os interlocutores de Räde em Belém foram os professores Aloysio da Costa Chaves (Reitor da UFPA, à época), José Seixas Laurenço, Herberto Tocantins Maltez e José Haroldo da Silva Sá 12.

Sobre o Laboratório de Física, Räde registrou que existiam salas mobiliadas para se instalar os equipamentos (cf. Fig. S6 - Material Suplementar), com a necessidade de pequenas adaptações, destacando, no entanto, a falta de oficinas mecânica, eletrônica e de fabricação de vidro, tendo o Reitor da UFPA se comprometido a providenciar estas oficinas. Räde ainda registrou que na primavera do ano seguinte, dois professores de física da UFPA seriam enviados para Jülich [12].

Os professores do Departamento de Física da UFPA Orlando José Carvalho de Moura e Leopoldino dos Santos Ferreira foram escolhidos para ir à Alemanha, no primeiro semestre de 1973, realizar um estágio no KFA, onde receberam instruções sobre equipamentos de um Curso Experimental em Física (cf. Fig. S7 - Material Suplementar) 13 15]. Aloysio Chaves havia originalmente solicitado a José Maria Filardo Bassalo (também professor de Física da UFPA) que fosse realizar este estágio na Alemanha, mas este preferiu não ir, indicando para esta tarefa Moura e Ferreira [16]. 6

Durante este estágio no exterior, Moura e Ferreira participaram de atividades experimentais envolvendo eletrônica, física moderna, técnicas de vácuo, holografia e baixas temperaturas. Nos Institutos de Eletrônica e de Físico-Química do KFA, em Jülich, realizaram, entre outros, experimentos com circuitos eletrônicos, diodos emissores de luz, instrumentos digitais, linhas de emissão, espectrômetro de massa, experimentos de Millikan, Compton, Franck-Hertz, Laue-Bragg. Os hologramas foram obtidos com o auxílio de um obturador especial, em um ambiente especialmente preparado para minimizar vibrações, tendo sido utilizado um espectrômetro do tipo de Michelson-Morley para detectar eventuais vibrações. Foram também realizadas experiências com Nitrogênio líquido 17]. Os experimentos utilizando técnicas de vácuo foram realizados nos Laboratórios da Leybold, em Colônia, e incluíram a obtenção de alto vácuo, técnicas em pressão muito baixa, evaporação de metais, bombas

\footnotetext{
${ }^{5}$ H. S. Räde foi um dos co-autores (da Parte C) do Curso Experimental em Física de Jülich, do qual trataremos em seguida.

6 Informação obtida em entrevistas concedidas por José Maria Filardo Bassalo a Luís C. B. Crispino, e mensagens eletrônicas entre ambos.
}

centrífugas, bombas de difusão e de absorção e medidas do efeito Zeeman 17].7

Em seu relatório ao CNPq, datado de 28 de junho de 1973, Moura registrou 17:

"Um dos problemas urgentes que defrontam hoje as universidades brasileiras é o do ensino da graduação. À medida que a pesquisa se torna cada vez mais absorvente para os professores, tem entrado em operação, com relativa frequência, uma sutil desvalorização do processo de ensino. Além disso, em muitos outros campos, a variação do conteúdo e da estrutura do conhecimento, uma consequênca da pesquisa, criou uma necessária e urgente revisão curricular [sic.]. Como é do nosso conhecimento, isto é especialmente uma grande verdade no campo específico da Ciência Física.

Moura finaliza este seu relatório escrevendo [17:

"O Curso que presentemente somos conhecedores de pormenores, incorporou os esforços de muitos físicos que trabalharam ou trabalham em áreas pioneiras de pesquisa, e foi afortunado por contar com o apoio do governo. O Curso precisa ser testado na Amazônia, tal como já o foi em outras universidades brasileiras, que dispõem do mesmo equipamento, durante um período de vários semestres, por alunos do primeiro biênio em Física. O Curso representa um pronunciado avanço educacional e espero que seja proveitoso para a região. O mais satisfatório será, talvez, quando despertarmos um vivo interesse no ensino de graduação, evidenciando-se com um número substancial de membros do corpo docente da Universidade, dispostos a participar desse programa de aperfeiçoamento curricular de Laboratório."

Ainda em 1973, após o retorno de Moura e Ferreira, os equipamentos do KFA chegaram em Belém, em um avião de carga 18.8 Cerca de uma semana depois, 9 chegaram alguns dos co-autores do Curso Experimental em Física do KFA, de Jülich [13 15], conforme registrou a imprensa paraense [19]:10

"Desde segunda-feira [23 de julho de 1973] se encontram em Belém quatro técnicos alemães contratados pela Universidade Federal do Pará para a montagem e treinamento dos professores do Laboratório de Física, no Núcleo Pioneiro da Universdidade (cf. Fig. S8 - Material Suplementar). São os técnicos Paul-Gerhard [Manteuffel] [20], Hans Peter Eulenberg, Ernst-Peter Röth e Ulrich [Herbert] Kurz, todos do Centro de Pesquisa de Julich - West Germany. Amanhã [26 de junho de 1973]

\footnotetext{
7 Moura e Ferreira também tiveram a oportunidade de conhecer vários outros institutos constituintes do KFA, incluindo o Instituto de Física do Estado Sólido, o Instituto de Física Nuclear e de Segurança à Radiação, o Instituto de Física de Plasma e o Instituto de Biofísica, além de visitarem reatores nucleares e as oficinas do KFA.

${ }^{8}$ Informação obtida em mensagens eletrônicas de Ernst-Peter Röth para Luís C. B. Crispino.

${ }^{9}$ Informação obtida em mensagens eletrônicas de Ernst-Peter Röth para Luís C. B. Crispino.

${ }^{10}$ Segundo Ernst-Peter Röth naquela ocasião já havia na UFPA alguns experimentos de física doados pela então Alemanha Oriental (Deutsche Demokratische Republik - DDR), incluindo um espectrômetro ótico.
} 
estará chegando a Belém o técnico [Willi] Rolnik [20], completando a equipe de cinco técnicos encarregados de montar o laboratório.

[...] Este se divide em peças para a parte de física básica e outra parte para física moderna. A parte de física básica se subdivide em mecânica, ótica, termodinâmica, eletricidade, magnetismo e acústica. A parte de física moderna inclui radioatividade, microondas, eletrônica, espectroscopia, bombeamento ótico e técnica de vácuo.

[...] Após o período de 4 semanas previsto para a montagem dos equipamentos, uma outra fase será de treinamento dos professores do Departamento de Física da Universidade. Esse treinamento, na fase inicial, será feito pelos próprios técnicos alemães, posteriormente, já na fase de funcionamento efetivo, a supervisão ficará com os professores Leopoldino Ferreira e Orlando Moura, pelo tempo aproximado 6 meses. De acordo com as datas aproximadas fornecidas pelo professor Leopoldino Ferreira, o Laboratório de Física da Universidade Federal do Pará só estará funcionando efetivamente no próximo ano."

Na chegada dos equipamentos, se fez presente o Reitor da UFPA, Clóvis Cunha da Gama Malcher [19] (cf. Fig. S9 - Material Suplementar), que havia sido empossado em Brasília em 02 de julho de 1973 [21], sucedendo Aloyso Chaves neste cargo.

Conforme anunciado na imprensa, durante o período que passaram em Belém, os enviados do KFA removeram os equipamentos de suas embalagens e os montaram, sendo auxiliados por alguns membros do Departamento de Física da UFPA, incluindo Moura, Ferreira e Fernando Medeiros Vieira, sendo este último o Chefe do Laboratório de Física da UFPA, à época. Também participaram destas atividades Hamilton Nazareno Ramos Schaefer, Ivo Vencato e Marco Antônio Stanford de Vasconcelos (cf. Fig. 11, ${ }^{11}$ da Universidade Federal de Santa Catarina (UFSC), e Almir Américo dos Santos (cf. Fig. S10 Material Suplementar), representando o CNPq naquele período de instalação dos equipamentos em Belém. ${ }^{12}$ Schaefer (Vice-Reitor da UFSC, à época), Vasconcelos e Vencato vieram a Belém para acompanhar a instalação dos experimentos alemães que também seriam implementados na UFSC, em seguida.

A equipe do KFA ministrou um curso intensivo sobre o uso dos equipamentos, direcionado especialmente a alguns auxiliares de ensino da UFPA. ${ }^{13} \mathrm{Na}$ Fig. 2 vemos Carmelina Nobukó Kobayashi, José Maria Costa

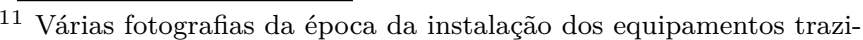
dos da Alemanha foram localizadas no acervo da UFPA por Manoel Januário da Silva Neto, professor da Faculdade de Física da UFPA.

${ }^{12}$ Informação obtida em mensagens eletrônicas de Marco Antônio Stanford de Vasconcelos, Ivo Vencato e Hamilton Nazareno Ramos Schaefer para Luís C. B. Crispino.

13 De acordo com Luiz Cancela, este curso intensivo não pôde ser plenamente aproveitado, uma vez que a maioria daqueles que assistiram o curso não tinha fluência em língua inglesa, idioma utilizado na ocasião para comunicação entre os estrangeiros e os brasileiros.
}

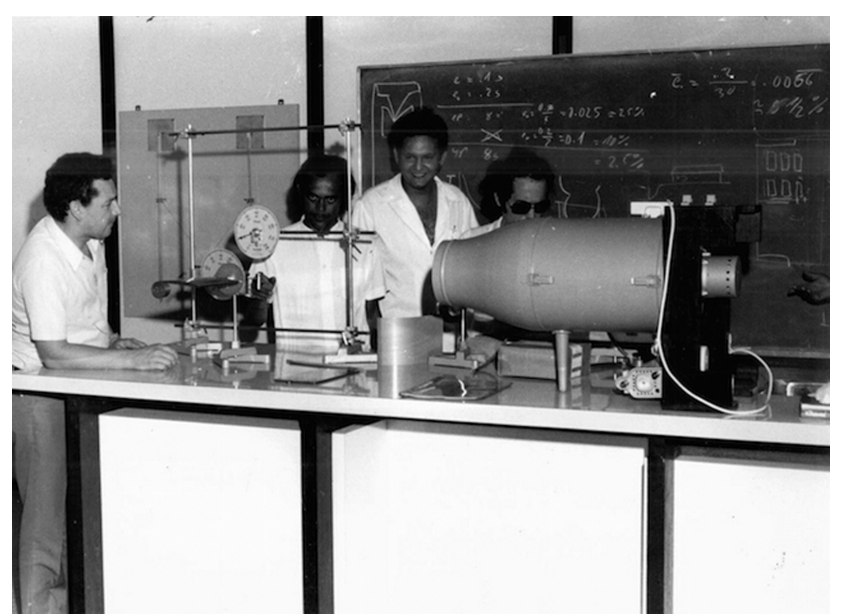

Figura 1: Realização de experimentos no Laboratório de Física da UFPA, utilizando o Túnel de Vento com seus acessórios, que foi um dos equipamentos enviados pela Alemanha em 1973. Este equipamento, que permite a realização de vários experimentos sobre a aerodinâmica de objetos expostos a um fluxo de ar, foi bastante utilizado nas décadas seguintes, até que a falta de peças de reposição impedisse que continuasse a ser utilizado. Da esquerda para a direita, figuram: Hamilton Nazareno Ramos Schaefer, Curt Rebello Sequeira, Orlando José Carvalho de Moura e Marco Antônio Stanford de Vasconcelos. Acervo da UFPA.

de Souza, Lindalva do Carmo Ferreira ${ }^{14}$ e Luiz Sérgio Guimarães Cancela, ${ }^{15}$ à época auxiliares de ensino do Departamento de Física da UFPA, juntamente com o técnico de Laboratório Juarez Magalhães de Vasconcelos, participando, em 1973, do curso de capacitação para a utilização dos equipamentos alemães de laboratório. ${ }^{16}$

A inauguração do Laboratório de Física com os experimentos alemães em funcionamento, ocorreu em 21 de agosto de $197320,22,23$. Sobre este episódio registrou o jornal de Belém "Folha do Norte" [22]:

"[...] segundo o Reitor Clóvis Malcher [o Laboratório] 'permite a formação profissional do professor, por ser um dos melhores do mundo' o que lhe dava a oportunidade de afirmar 'que doravante nossos mestres não precisam mais ir ao Sul em busca de aprimoramento'.

O laboratório doado pela Alemanha veio para a UFPA, em decorrência de um convênio com o Governo Brasileiro para ajuda mútua técnico-científica (cf. Fig. S11 Material Suplementar). [...]

${ }^{14}$ Lindalva do Carmo Ferreira fez parte da segunda turma de alunos de Física da UFPA, tendo ingressado no Curso de Licenciatura em Física no ano de 1967, e concluído em 1970 5].

15 De acordo com Luiz Cancela, no ano de 1966, apenas dois candidatos inscreveram-se para o Curso de Física da UFPA e, por este número insuficiente, o vestibular daquele ano para o Curso de Física foi cancelado na instituição paraense. Um destes dois candidatos, o próprio Luiz Cancela, viu-se obrigado e escolher um outro Curso, formando-se em Matemática em 1969, e ingressando na UFPA no ano seguinte como auxiliar de ensino.

16 Informação obtida em entrevistas concedidas por Carmelina Nobukó Kobayashi Luiz Sérgio Guimarães Cancela, Lindalva do Carmo Ferreira, e José Maria Costa de Souza; a Luís C. B. Crispino, e mensagens eletrônicas. 


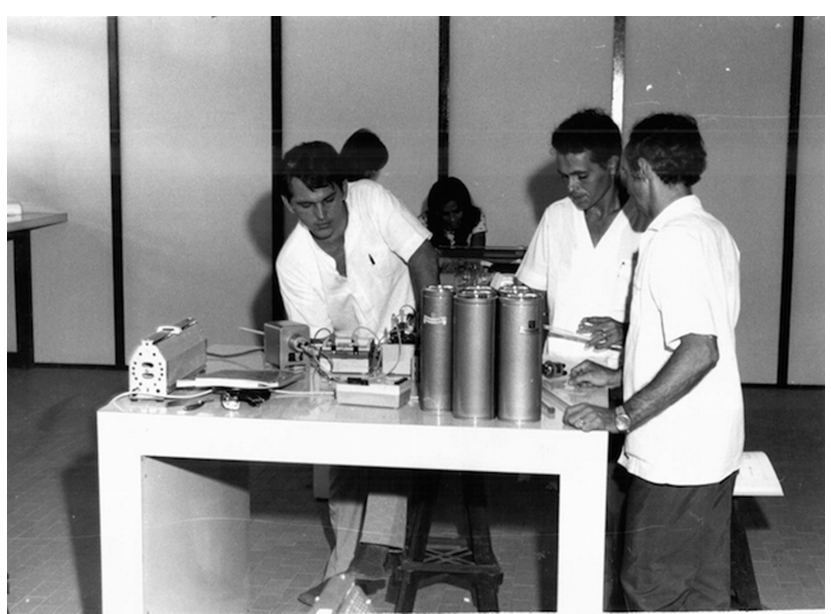

Figura 2: Grupo de auxiliares de ensino sendo capacitado para a utilização dos equipamentos alemães no Laboratório de Física da UFPA, em 1973. Da esquerda para a direita vemos Luiz Sérgio Guimarães Cancela, Carmelina Nobukó Kobayashi, Lindalva do Carmo Ferreira, José Maria Costa de Souza e Juarez Magalhães de Vasconcelos, técnico de Laboratório. Sobre a bancada da frente vemos equipamentos relacionados com experimentos de Calor e Eletricidade. Acervo da UFPA.

A fita de inauguração foi desmanchada pelo Ministro da Educação (cf. Fig. S12 - Material Suplementar) e pelo Consul da Alemanha. Depois, os professores Vencato Marconi [sic.] e Marco Antonio Vasconcelos mostraram a todos os presentes o funcionamento do Laboratório de Física. [...]"

Além do Ministro da Educação e Cultura, Jarbas Passarinho; do Vice-Governador do Estado do Pará, Newton Barreira; do Reitor e ex-Reitor da UFPA, Cóvis Malcher e Aloysio Chaves, respectivamente; do Cônsul da Alemanha para o Norte e Nordeste do Brasil, Peter Bensch; do representante do CNPq, Coronel Sebastião Furtado; entre muitos outros; nesta inauguração estiveram presentes o Diretor da Casa de Estudos Germânicos da UFPA, Konrad Ackermannn, a equipe alemã do KFA, e os professores do Departamento de Física da UFPA, Leopoldino Ferreira e Orlando Moura 20,23 (cf. Figs. 3 S13 e S14 Material Suplementar).

O Cônsul da Alemanha declarou que "a ajuda prestada por seu país ao Brasil é modesta, valendo, entretanto, o entrosamento cultural, técnico e científico entre os dois países, pois a cada dia a Alemanha vê o Brasil se tornar mais forte, daí considerar privilégio comungar a Alemanha dessa arrancada de desenvolvimento do Brasil" 23].

Já o representante do CNPq lembrou "a ajuda alemã aos projetos educacionais brasileiros, não só no Pará, como em São Paulo, Rio, Brasília e outros Estados, e que o Conselho Nacional de Pesquisa confia plenamente na capacidade dos técnicos paraenses" 23.

Passado cerca de um ano desta inauguração, em meados do ano de 1974, o mineralogista alemão Thomas Scheller iniciou sua carreira na UFPA como professor visitante, atuando em um convênio entre a Universidade de Erlangen, da Alemanha e o Núcleo de Ciências Geofí-

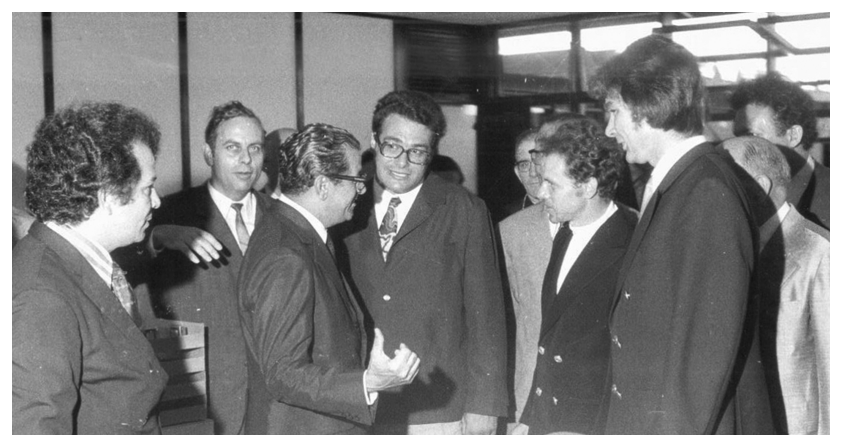

Figura 3: Foto tirada em 21 de agosto de 1973, no Laboratório de Física da UFPA, figurando da esquerda para a direita: Leopoldino Ferreira, Willi Rolnik, Jarbas Passarinho, Hans Peter Eulenberg, Cônego Ápio Campos, Ulrich Herbert Kurz, PaulGerhard Manteuffel, Ernst-Peter Röth, Clóvis Malcher e Orlando Moura, Acervo da UFPA.

sicas e Geológicas na UFPA, no âmbito de um projeto de cooperação internacional, coordenado do lado brasileiro por José Seixas Lourenço. ${ }^{17}$ Do lado alemão este convênio foi financiado pela KFA Jülich, a mesma instituição responsável pelos equipamentos alemães enviados para o Laboratório de Física da UFPA. Ficou então decidido que Scheller dedicaria parte de seu tempo para a manutenção destes equipamentos. 18

No segundo semestre de 1974, Scheller recebeu a visita na UFPA de Ulrich Kurz, engenheiro da KFA que retornou a Belém após sua vinda inicial para instalação dos equipamentos no ano anterior. Scheller e Kurz visitaram naquele mesmo ano os laboratórios brasileiros das Universidades Federais de Brasília, Santa Catarina e São Carlos, que também tinham recebido equipamentos da mesma instituição alemã. Kurz retornou para a Alemanha em seguida. 19

Os professores da Universidade de Brasília, Carlos Alberto da Silva Lima, Ademar Silveira Aragão e Francisco Rogério Fontenele Aragão, foram convidados para ministrar no Laboratório de Física da UFPA, juntamente com Scheller, um treinamento para a utilização dos experimentos alemães, o que ocorreu no início do ano de 1975. 20

Os professores Carlos Lima, Thomas Scheller, Ademar Aragão e Francisco Aragão elaboraram um Roteiro de Experiências (cf. Fig. S15 - Material Suplementar) [24], que foi utilizado durante o Curso de Física Experimental (como foi oficialmente denominado o treinamento pelo Departamento de Física da UFPA), com o financiamento da própria UFPA e da Coordenação de Aperfeiçoamento de Pessoal de Ensino Superior (CAPES). Este Curso foi dividido nas unidades de (i) Física Geral I, (ii) Física

\footnotetext{
17 Informação obtida em mensagens eletrônicas de Thomas Scheller para Luís C. B. Crispino.

${ }^{18}$ Informação obtida em mensagens eletrônicas de Thomas Scheller para Luís C. B. Crispino.

${ }^{19}$ Informação obtida em mensagens eletrônicas de Thomas Scheller para Luís C. B. Crispino.

${ }^{20}$ Informação obtida em mensagens eletrônicas de Thomas Scheller e Carlos Alberto da Silva Lima para Luís C. B. Crispino.
} 
Geral II, (iii) Mecânica, (iv) Eletricidade e Magnetismo, e (v) Vibrações e Ondas. $21{ }^{22}$

Nos meses que se sucederam, Scheller participou de aulas práticas no Laboratório de Física da UFPA, realizando manutenção dos equipamentos e orientando monitores. 23

Uma das principais dificuldades relacionadas à utilização dos equipamentos alemães consistia no fato de que os manuais originais das experiências associadas a estes equipamentos [13 15] (cf. Fig S8 - Material Suplementar) estavam em língua inglesa.

Estes manuais, em uma de suas páginas iniciais, continham impresso o seguinte texto:

"Os experimentos contidos neste programa foram planejados e testados por cientistas do KFA Jülich GmbH, Alemanha Ocidental. Eles englobam uma edição revisada e ampliada de um curso doado em 1972 pelo Ministério da Educação da Alemanha para algumas universidades brasileiras." 2425

Juntamente com Carmelina Kobayashi, Scheller iniciou a tradução e adaptação para o português destes manuais de experimentos. Este trabalho foi interrompido em junho de 1977, com o retorno de Scheller para a Alemanha. 26 27

Após sua volta a Belém, em 1973, retornando do estágio na Alemanha, Orlando Moura e Leopoldino Ferreira também auxiliaram na montagem de alguns dos equipamentos alemães, e compartilharam com seus colegas e alunos da UFPA o que aprenderam na Alemanha. Moura ficou responsável pelo Laboratório de Física Moderna e Ferreira pelo Laboratório de Radiações do Departamento de Física da UFPA. Por vários anos, até sua aposentadoria, ministraram, respectivamente, as disciplinas "Física Moderna" e "Física Nuclear" para os alunos dos Cursos de Física da UFPA.

Entre os equipamentos de Física Moderna trazidos da Alemanha para a UFPA na década de 1970, alguns ainda estão atualmente em operação, como é o caso do experimento para verificação da relação carga-massa do elétron (Fig. S16 - Material Suplementar) e os tubos com gases

${ }^{21}$ Informação obtida em entrevistas concedidas por Carlos Alberto da Silva Lima a Luís C. B. Crispino, e mensagens eletrônicas entre ambos.

${ }^{22}$ Entre as pessoas que assistiram este Curso, na qualidade de estudantes de Licenciatura em Física, podemos citar José Luiz Magalhães Lopes e Victor Façanha Serra.

${ }^{23}$ Informação obtida em mensagens eletrônicas de Thomas Scheller para Luís C. B. Crispino.

24 Tradução livre do texto original em inglês: The experiments contained in this program were planed and tested by scientists of the KFA Jülich GmbH, West Germany. They comprise a revised and enlarged edition of a course which was donated in 1972 by the German Ministry of Education to some Brazilian universities.

25 Os professores contemporâneos a este episódio costumavam mencionar que estes equipamentos teriam sido enviados pela Alemanha em troca de café brasileiro.

26 Thomas Scheller retornou ao Brasil alguns anos depois, tendo reingressado na UFPA como professor efetivo no ano de 1982, permanecendo na instituiçao como professor até o ano de 2013, quando se aposentou.

${ }^{27}$ Informação obtida em mensagens eletrônicas de Thomas Scheller para Luís C. B. Crispino. acoplados à Bobina de Ruhmkorff (Fig. S17 - Material Suplementar). Estes dois equipamentos são utilizados até hoje em atividades da disciplina "Laboratório Especial" dos Cursos de Graduação em Física da UFPA.

Com o passar dos anos, devido principalmente à dificuldade de obtenção de peças de reposição, grande parte dos equipamentos trazidos da Alemanha na década de 1970 deixou de ser utilizada nas aulas práticas de física da UFPA. 28

No ano de 2017, por ocasião da celebração dos 60 anos da UFPA, foi realizada uma exposição de alguns dos experimentos que contituíram o Laboratório de Física da instituição na década de 1970. Nossa intenção para o futuro é que estes equipamentos de importância histórica para a UFPA sejam colocados à mostra para o público em um mobiliário apropriado, acondicionado em uma sala destinada a este fim no Laboratório de Física-Ensino da UFPA, ou em outro local, seguindo o exemplo de outras universidades mundo afora, como é o caso, por exemplo, do Dipartimento di Fisica / Istituto Fisico Guglielmo Marconi da Universidade La Sapienza, em Roma, na Itália [29], ou da coleção de instrumentos científicos e didácticos de física do Museu da Ciência da Universidade de Coimbra, em Portugal [30].

Neste artigo tratamos da instalação do Laboratório de Física da UFPA. A existência de peças históricas ainda em funcionamento do acervo deste laboratório da UFPA, bem como de um conjunto representativo de fotografias desta etapa do ensino de física no Norte do Brasil, nos serviram de motivação adicional para a realização deste artigo. Acreditamos que os fatos relatados aqui guardem semelhança com a história de laboratórios de física de outras universidades federais brasileiras. 29

\section{Agradecimentos}

A recomposição dos fatos que constituíram a gênese do Laboratório de Física da UFPA só foi possível devido à colaboração de pessoas de diversas instituições no Brasil e no exterior. Somos especialmente gratos a Alcyr Bóris de Souza Meira, Brígida Ramati Pereira da Rocha, Carlos Alberto da Silva Lima, Carmelina Nobukó Kobayashi, Ernst-Peter Röth, Fernando Medeiros Vieira, Hamilton Nazareno Ramos Schaefer, Ivo Vencato, José Luiz Magalhães Lopes, José Maria Costa de Souza, José

\footnotetext{
28 Hoje o acervo do laboratório didático de física da UFPA é constituído principalmente por equipamentos construídos pelas empresas Phywe Systeme GmbH \& Co. KG 25], Centro Industrial de Equipamentos de Ensino e Pesquisa (CIDEPE) [26], Azeheb 27], e Ciência Prima 28].

29 Vale registrar aqui, em especial, a cessão de um dos equipamentos alemães, enviados pelo KFA, entre duas universidades que os receberam. A Universidade Federal do Rio Grande do Norte (UFRN) emprestou um eletromagneto, sem fonte de corrente, para a Universidade Federal de Pernambuco (UFPE), que também havia recebido um exemplar deste mesmo equipamento. Estes eletromagnetos foram utilizados nas primeiras pesquisas experimentais de Magnetismo em Pernambuco e ainda hoje são usados nos laboratórios de pesquisa da UFPE. Somos gratos a Liacir dos Santos Lucena e a Sergio Machado Rezende por estas informações.
} 
Maria Filardo Bassalo, José Seixas Lourenço, Liacir dos Santos Lucena, Lindalva do Carmo Ferreira, Luiz Sérgio Guimarães Cancela, Marco Antônio Stanford de Vasconcelos, Miguel Ayan Gaia, Sergio Machado Rezende e Thomas Scheller - que protagonizaram alguns dos fatos aqui relatados - por conversas, entrevistas e trocas de mensagens eletrônicas, e disponibilização de documentos que foram fundamentais para a recomposição da história de que trata este artigo. Somos também gratos a Basílio Baseia, Bernhard Mittermaier, Bernd-Alfred Rusinek, Candido Augusto Veloso Moura, Débora Peres Menezes, Igor José do Carmo Coimbra, Jadir Horácio Sarmento Pinto Júnior, Jens Dreyer, João Furtado de Souza, José David Mangueira Viana, José Jerônimo de Alencar Alves, Juliana Martins Ferreira, Manoel Januário da Silva Neto, Marcelo Costa de Lima, Marcondes Lima da Costa, Petrus Agrippino de Alcantara Junior, Rômulo Simões Angélica, Rubens de Paula Borges Cavalcante, Ruy Guilherme Castro de Almeida, Sanclayton Geraldo Carneiro Moreira, Wanda Pereira Ignacio, Werner Hermann Walter Truckenbrodt e Zínia de Aquino Valente. Agradecemos também a Carlos Eduardo Pires Martins e Manoel Januario da Silva Neto pela realização de fotografias que ajudaram no levantamento do acervo do Laboratório de Física-Ensino da UFPA e na composição deste artigo, e a toda a equipe do Laboratório de Demonstrações da UFPA [31], pelo escaneamento de documentos associados à instalação e inauguração dos equipamentos do Laboratório de Física-Ensino da UFPA. Agradecemos ainda a Maria Ieda Raposo de Moura e Vânia Raposo de Moura, viúva e filha, respectivamente, de Orlando José Carvalho de Moura, bem como a Benedita Maria Costa, viúva de Leopoldino dos Santos Ferreira, pela ajuda na localização de documentos relacionados ao assunto deste artigo. Somos gratos também a Simone Maria Neno Silva, e à equipe da Editora da UFPA, pela organização de uma exposição com os experimentos históricos do Laboratório de Física da UFPA, durante a edição de 2017 da Feira Pan-Amazônica do Livro, o que impulsionou os autores para a realização deste artigo. L. C. agradece a Ângela Burlamaqui Klautau e José Maria Filardo Bassalo, por comentários e sugestões a este trabalho. Somos gratos às diferentes composições da Administração Superior da UFPA que, ao longo dos anos, permitiram não só a instalação do Laboratório de Física da instituição na década de 1970, mas que também têm dado o apoio necessário para os Cursos de Física da UFPA. Registramos também nossos agradecimentos aos servidores técnico-administrativos do Laboratório de Física-Ensino da UFPA, em particular a Joaquim Gomes da Silva e a Paulo Sérgio da Silva Corrêa; bem como a Nelma Silva Milhomem e a Ranulfo Figueiredo Campos, da Biblioteca Pública do Pará, em Belém. Gostaríamos também de agradecer o apoio do Conselho Nacional de Desenvolvimento Científico e Tecnológico (CNPq) e da da Coordenação de Aperfeiçoamento de Pessoal de Nível Superior (CAPES).

\section{Material Suplementar}

O seguinte material suplementar está disponível online: Figura S1 - Hemisférios de Magdeburgo. Acervo do Laboratório de Física-Ensino da UFPA. Fotografia de Carlos E. P. Martins.

Figura S2 - Centrífuga. Acervo do Laboratório de Física-Ensino da UFPA. Fotografia de Carlos E. P. Martins.

Figura S3 - Câmara Escura comercializada pela firma Otto Bender, de São Paulo. Acervo do Laboratório de Física-Ensino da UFPA. Fotografia de Carlos E. P. Martins.

Figura S4 - Bobina de Ruhmkorff comercializada pela firma Otto Bender, de São Paulo. Acervo do Museu Interativo da Física da UFPA. Fotografia de Luís C. B. Crispino.

Figura S5 - Experimento ilustrativo de Motor a Combustão comercializado pela firma Otto Bender, de São Paulo. No detalhe (canto superior direito), encontram-se destacadas a logomarca da firma Otto Bender (à esquerda) e a etiqueta de tombamento de número 419, da então Universidade do Pará (à direita). Acervo do Museu Interativo da Física da UFPA. Fotografia de Luís C. B. Crispino.

Figura S6 - Imagem de uma das salas de aula do Laboratório de Física da UFPA, constante no relatório alemão de 1972, de autoria de H. S. Räde [14]. Cortesia do KFA, Jülich, Alemanha.

Figura S7 - Capa da Parte A do Curso Experimental em Física do KFA Jülich, contendo instruções acerca dos equipamentos de Mecânica, Acústica, Termodinâmica e Ótica trazidos da Alemanha [15]. Acervo do Laboratório de Física-Ensino da UFPA.

Figura S8 - Imagem publicada na edição de 25 de julho de 1973, do jornal de Belém "A Província do Pará", com a legenda: "Os técnicos que vieram montar o Laboratório da UFPA"[24]. Da esquerda para a direita, vemos: Hans Peter Eulenberg, Ernst-Peter Röth e Paul-Gerhard Manteuffel. Fotógrafo desconhecido. Cortesia da Biblioteca Pública do Pará.

Figura S9 - Apresentação de alguns dos equipamentos alemães ao Reitor da UFPA, à época, Clóvis Cunha da Gama Malcher, no Laboratório de Física. Da esquerda para a direita figuram Ulrich Herbet Kurz, Clóvis Malcher, Ernst-Peter Röth e Leopoldino dos Santos Ferreira. Acervo da UFPA.

Figura S10 - Preparação para a realização de alguns dos experimentos com os equipamentos enviados pela Alemanha em 1973, para o Laboratório de Física da UFPA. À esquerda vemos Almir Américo dos Santos, do $\mathrm{CNPq}$, e à direita vemos Fernando Medeiros Vieira, Chefe do Laboratório de Física da UFPA, à época [7]. Acervo da UFPA.

Figura S11 - Etiqueta da Universidade Federal do Pará afixada em um dos equipamentos alemães, associados ao convênio entre o CNPq e o KFA. Vale ressaltar que todas as etiquetas alusivas a este convênio que localizamos no atual Laboratório de Física-Ensino da UFPA não 
possuem gravação de número, como é o caso da que exibimos aqui. Fotografia de Victor Façanha Serra.

Figura S12 - Foto no momento do desmanche da fita de inauguração do Laboratório de Física da UFPA, em 21 de agosto de 1973. Da esquerda para a direita vemos: o Ministro da Educação e Cultura Jarbas Passarinho, Ernst-Peter Röth, Ulrich Kurz, e (parcialmente) o Cônsul da Alemanha Peter Bensch. Acervo da UFPA.

Figura S13 - Apresentação dos equipamentos alemães ao Ministro Jarbas Passarinho, no Laboratório de Física, em 21 de agosto de 1973. Acervo da UFPA.

Figura S14 - Cena no dia da inauguração dos equipamentos alemães, no Laboratório de Física da UFPA. Acervo da UFPA.

Figura S15 - Capa do Roteiro de Experiências elaborado por Carlos Alberto da Silva Lima, Thomas Scheller, Ademar Silveira Aragão e Francisco Rogério Fontenele Aragão [24]. Cortesia de Carlos Alberto Lima.

Figura S16 - Experimento para verificação da relação carga-massa do elétron, trazido da Alemanha para a UFPA na década de 1970. Acervo do Laboratório de Física-Ensino da UFPA. Fotografia de Victor Façanha Serra.

Figura S17 - Tubos com gases e Bobina de Ruhmkorff, ainda hoje em operação, trazidos da Alemanha para a UFPA na década de 1970. Acervo do Laboratório de Física-Ensino da UFPA. Fotografia de Victor Façanha Serra.

\section{Referências}

[1] BRASIL. Lei No. 3191, de 2 de julho de 1957.Brasília, 1957. Disponível em http://www2.camara.leg.br/ legin/fed/lei/1950-1959/lei-3191-2-julho-1957354771-norma-pl.html/

[2] E. J. O. Fontes. UFPA 50 Anos - Histórias e memórias (Editora Universitária UFPA, Belém, 2007).

[3] J. M. F. Bassalo, Revista Brasileira de Ensino de Física 17, 152 (1995).

[4] W. N. de Lima, P. de T. S. Alencar e R. dos S. Barbosa. in: Anais do Simpósio sobre a História da Ciência e da Tecnologia no Pará. Tomo I (Gráfica e Editora Universitária, UFPA, Belém, 1985), editado por C. F. R. Beckmann, J. M. F. Bassalo, C. C. Bassalo, M. G. Maltez, H. T. Maltez, P. de T. S. Alencar e W. N. de Lima. (Gráfica e Editora Universitária, UFPA, Belém, 1985), p. 113-179.

[5] R. G. C. de Almeida. Engenheiros e Matemáticos na História do Ensino de Física no Estado do Pará (19311970). Tese de Doutorado, Universidade de São Paulo, São Paulo (2006).

[6] G. P. dos S. Cavalcante. in: Anais do Simpósio sobre a História da Ciência e da Tecnologia no Pará. Tomo I, Belém, 1985, editado por C. F. R. Beckmann, J. M. F. Bassalo, C. C. Bassalo, M. G. Maltez, H. T. Maltez, P. de T. S. Alencar e W. N. de Lima (Gráfica e Editora Universitária, UFPA, Belém, 1985), p. 321-343.

[7] R. G. C. de Almeida. A História do Ensino de Física no Estado do Pará (1904-1961). Dissertação de Mestrado, Universidade Federal do Pará, Pará (1997).
[8] Universidade inaugura novas salas de aula para o progresso do ensino. O Liberal, Belém, 23 de março de 1971.

[9] Palavra do Reitor na Aula Inaugural, Folha do Norte, Belém, 23 de março de 1971.

[10] Um Brinde à Arrancada da Reforma. A Província do Pará, Belém, 24 de março de 1971.

[11] Aula de Jarbas para calouros será amanhã. Folha do Norte, Belém, 21 de março de 1971.

[12] H. S. Räde. Bericht über eine Dienstreise nach Brasilien vom 23.11.72- 17.12.72 im Auftrage des Internationalen Br̈os der KFA (em alemão), 1972.

[13] U. Kurz, E.-P. Röth e P. Tiedemann. An Experimental Course in Physics - Part A. Mechanics, Acoustics, Thermodynamics, Optics. Kernforschungsanlage Jülich, Jülich, 1972.

[14] H. P. Eulenberg e P. G. Manteuffel. An Experimental Course in Physics - Part B. Electricity and Magnetism Kernforschungsanlage Jülich, Jülich, 1972.

[15] H. P. Eulenberg, H. S. Räde, W. Rolnik, e E. P. Röth. An Experimental Course in Physics - Part C. Introduction to Various Methods of Modern Physics. Kernforschungsanlage Jülich, Jülich, 1972.

[16] http://www.bassalo.com.br/, acessado em 26/03/2017.

[17] O. J. C. de Moura. Relatório de Pesquisa sobre estágio no KernForschungsAnlage (KFA), 1973.

[18] Hp. Eulenberg, U. Kurz, P.-G. Manteuffel, W. Rolnik and E.-P. Röth. Bericht über einen Aufenthalt in Brasilien vom 23 Juli bis zum 29, KFA, 1973.

[19] Universidade Monta o Laboratório de Física. A Província do Pará, Belém, 25 de julho de 1973.

[20] Jarbas inaugurou o Moderno Laboratório de Física da UFPA. A Província do Pará, Belém, 22 de agosto de 1973.

[21] Reitor foi empossado em Brasília. O Liberal, Belém, 03 de julho de 1973.

[22] Ensino para toda a cidade. Folha do Norte, Belém, 22 de agosto de 1973.

[23] Equipamento de Física da UFPA é dos mais modernos. O Liberal, Belém, 22 de agosto de 1973.

[24] C. A. Lima, T. Scheller, A. S. Aragão e F. R. Aragão. Roteiro de Experiências, Universidade Federal do Pará, 1975.

[25] http://www.phywe-systeme.com/, acessado em 26/03/2017.

[26] http://www.cidepe.com.br, acessado em 26/03/2017.

[27] http://azeheb.com.br, acessado em 26/03/2017.

[28] http://www.cienciaprima.com.br acessado em 26/03/2017.

[29] http://www.phys.uniroma1.it/fisica/, acessado em 26/03/2017.

[30] http://www.museudaciencia.org/, acessado em $26 / 03 / 2017$.

[31] J. Caldas e L. C. B. Crispino. Revista Brasileira de Ensino de Física, 39, e2309 (2017). 